\title{
A Channel Allocation Algorithm Based on Demand in Cognitive Wireless Mesh Networks
}

\author{
Tingrui Pei, Jing Li and Zhetao Li \\ College of Information Engineering \\ Xiangtan University, Xiangtan 411105 , China \\ Key Laboratory of Intelligent Computing \& Information \\ Processing of Ministery of Education, Xiangtan University
}

\begin{abstract}
We proposed a channel allocation algorithm, which was a modification of graph theory and based on demand to optimize the performance of cognitive wireless Mesh network. The key idea of the algorithm was to design a proportion coefficient of actual flow to demand flow, and then cognitive users with the prior proportion could access their optimal channels. The results of simulation show that the proposed algorithm improves the throughput and fairness of network.
\end{abstract}

Keywords-cognitive radio; wireless Mesh network; demand proportion; fairnessork.

\section{INTRODUCTION}

Wireless Mesh Network (WMN for short) is a multi-pointto-multi-point wireless network with high speed. Compared with the traditional wireless network, it has many advantages such as agility, high network coverage and large network capacity and so on.

However, with the rapid development of wireless communication technology and the increasing demand for wireless communication businesses, wireless network resources are scarcer and inadequate. Spectrum management mode and spectrum allocation scheme are the main reasons. According to the traditional fixed spectrum allocation scheme, the government will allocate a certain spectrum for each radio service, which is only for authorized users. In fact, however, the spectrum has not been fully utilized. According to the data from FCC, the variation range of spectrum allocation and utilization in time and space is $15 \% \sim 85 \%$. In 1999, Dr. Joseph Mitola put forward the cognitive radio (CR), and he detailed its concept in his doctoral dissertation ${ }^{[1]}$. Cognitive radio (CR) is a revolutionary technology, with the benefit of advanced spectrum sensing technology and dynamic channel allocation technology to realize the effective use of spectrum ${ }^{[2][3]}$. Authorized users in cognitive network are also called the primary users (PU), who are traditional radio users and authorized by frequency authorities to take legal use of certain frequency band. Cognitive users (also called SU), as a counterpart of PU, use temporarily unoccupied frequency band that authorized to PU. CR is now considered to be the best solution to the low utilization of wireless spectrum, and channel resources allocation is a key technology in cognitive radio.

This paper probes into the cognitive wireless mesh network (CWMN) channel resources allocation mechanism based on the combination of cognitive radio technology and wireless mesh network. At present, abundant researches have achieved in channel allocation. Das ${ }^{[4]}$ presented the optimization models for fixed channel assignment in WMN, only without presenting practical polynomial time algorithm, and the static model is obviously unsuitable for cognitive radio technology. Wang ${ }^{[5]}$ put forward an allocation algorithm based on list coloring, only without taking account of the differences of the channel benefit. Peng $^{[6]}$ displayed a color sensitive graph coloring (CSGC) algorithm and analyzed in detail the benefits and fairness of the spectrum allocation, only with complicated computation, on which basis. Liao $^{[7]}$ proposed a cognitive radio spectrum allocation algorithm in parallel and simplified the computation. $\mathrm{Liu}^{[8]}$ puts forward the maximal independent set based channel allocation algorithm, which to some extent, takes both the utilization and the fairness of the channel allocation into consideration, and reduces the convergence time. This algorithm is more suitable for the actual requirements of cognitive radio dynamic spectrum allocation, but it still owns the deficiency of not considering the differences of channel benefit and the own need of mesh network node. Therefore, this paper mainly proposed a new channel allocation mechanism to realize the performance ascension of the network.

\section{CWMN SYSTEM MODEL}

The structure of cognitive wireless mesh network can be divided into three types: backbone WMN, client Ad-hoc WMN and hybrid WMN, to meet the needs of both authorized and unauthorized application, while hybrid WMN is the one discussed in this paper. There are many factors to be considered about the CWMN channel allocation, such as interference, routing, connectivity, etc., which will directly affect the performance of the network, and through appropriate channel allocation algorithm, the network service quality can be optimized. In this paper we use the graph theory model to solve the problems emerging in the process of channel allocation.

Considering of a cognitive wireless mesh network, it is defined by a directed graph $\mathrm{G}(\mathrm{N}, \mathrm{M}, \mathrm{C})$. This model consists of several PU and $\mathrm{n}(\mathrm{n} \in \mathrm{N}) \mathrm{SU}$, sharing $\mathrm{m}(\mathrm{m} \in \mathrm{M})$ channels. $\mathrm{C}$ denotes constraint set hereinafter. Here, we assume that SU nodes can accurately perceive the radio environment and the information of all channels before transmitting the data.

The unified graph coloring model can be displayed as follow: 


\section{A. Leisure matrix}

Let $L=\left\{l_{\mathrm{n}, \mathrm{m}} \mid l_{\mathrm{n}, \mathrm{m}} \in\{0,1\}\right\}_{\mathrm{N} \times \mathrm{M}}, l_{\mathrm{nm}}=1$ characterizes channel $\mathrm{m}$ is available for $\mathrm{SU} \mathrm{n}$, on the other hand $l_{\mathrm{m}}=0$ represents unavailable, i.e. channel $\mathrm{m}$ couldn't be accessed by SU who is in the transmission range of the PU occupied the channel $\mathrm{m}$.

\section{B. Constraint matrix}

Let $C=\left\{C_{\mathrm{n}, \mathrm{m}} \mid C_{\mathrm{n}, \mathrm{m}} \in\{0,1\}\right\}_{\mathrm{N} \times \mathrm{N}}, C_{\mathrm{n}, \mathrm{k}}=1$ reflects that node $\mathrm{n}$ and node $\mathrm{k}$ would cause interference if they used the same channel simultaneously. Of cause, $c_{\mathrm{n}, \mathrm{k}}=0$ if $n=k$.

\section{Allocation matrix}

Let $A=\left\{a_{\mathrm{n}, \mathrm{m}} \mid a_{\mathrm{n}, \mathrm{m}} \in\{0,1\}\right\}_{\mathrm{N} \times \mathrm{M}}, a_{\mathrm{n}, \mathrm{m}}$ indicates the allocation state. Channel $\mathrm{m}$ has assigned to node $\mathrm{n}$ while $a_{\mathrm{n}, \mathrm{m}}=1$, specifically allocation matrix essences must satisfy the condition:

$$
a_{\mathrm{n}, \mathrm{m}} \times a_{\mathrm{k}, \mathrm{m}}=0 \quad \text { if } c_{\mathrm{n}, \mathrm{k}}=1
$$

\section{Benefit matrix}

Let $B=\left\{b_{\mathrm{n}, \mathrm{m}} \mid b_{\mathrm{n}, \mathrm{m}} \in\{0,1\}\right\}_{\mathrm{N} \times \mathrm{M}}, b_{\mathrm{n}, \mathrm{m}}$ describes the reward that $\mathrm{SU} n$ gains by successfully acquiring the available channel $\mathrm{m}$. Because system parameters in each network are different, nodes have different reward such as maximum throughput, channel utilization. We use channel capacity as the benefit parameter.

\section{IMPROVED CHANNEL ALLOCATION ALGORITHM}

Channel allocation algorithm which proposed in some literatures ${ }^{[7]}{ }^{[8]}$ only considered the benefits users gained while did not take their actual needs into account, which may lead to the excess bandwidth allocation for those in small demand while shortage in resources for those in need. In cognitive mesh network, imbalance load of nodes near the hotspots center is more serious. Therefore, this paper puts forward the allocation algorithm combined with demand, matching the allocation with users' demand. This paper aims to realize the maximized network benefits through on-demand channel allocation under the constraint of fairness coefficient and propose the Demand Proportion Fair Algorithm (hereinafter referred to as DPF).

In the cognitive network, in order to ensure the normal communication of PU, cognitive node use idle channel, and exit the channel as soon as the authorized node is back. Therefore, in the process of channel allocation we set the idle time of channel as one of the efficiency parameters to save the costs brought by the channel switch of cognitive node and the communication interruption. Another efficiency parameter is the channel transmission rate, corresponding to the transmission quality of the channel. With these two aspects combined, the node priority selection can achieve the channel access with maximum flow.
Based on Shannon theorem, SU' max-transmission rate can express as:

$$
b_{n, m}=\text { band }_{m} \log _{2}\left(1+K \frac{r_{n, m} \times H}{\text { band }_{m} \times \sigma_{0}^{2}}\right)
$$

We assume that the channel holding duration is $\mathrm{T}$ and the expected demand of node $\mathrm{n}$ shows $\varepsilon_{n}$ while the allocation process time is negligible. On the leisure channel set, the transmission time set can describe as follow:

$$
T=\left\{t_{n, m} \mid t_{\mathrm{n}, \mathrm{m}}=\varepsilon_{n} / b_{n, m}\right\}
$$

Scholars formulate the idle state of channel is a 0-1 Markov process and it is independent and identically distribute $\left.{ }^{[9][10}\right]$. The time $\mathrm{t}$ either channel $\mathrm{m}$ stays on state- 1 before turning to state- 0 obey the exponential distribution of parameter $\lambda_{\mathrm{m}}$. So the probability t exceed $t_{\mathrm{n}, \mathrm{m}}$ is represented by

$$
P_{\mathrm{n}, \mathrm{m}}\left(t \geq t_{\mathrm{n}, \mathrm{m}}\right)=1-\int_{-\infty}^{T} f_{\mathrm{m}}(t) d t=e^{-\lambda_{\mathrm{m}} \mathrm{t}_{\mathrm{n}, \mathrm{m}}}
$$

We use the probability value set as coloring label, then label $_{n}=\max P_{n, m}$. That means SU node $\mathrm{n}$ will choose the channel with max-value $P_{n, m}$.

Set the immediate flow of node $\mathrm{n}$ is $\theta_{n}$. Then corresponding proportional coefficient can be presented:

$$
\beta_{\mathrm{n}}=\theta_{n} / \varepsilon_{\mathrm{n}}
$$
follow:

The principle of fair constraint in our algorithm can state as

$$
\text { s.t }: \beta_{n} \leq \beta_{i}\left(\beta_{i}=\min \beta\right)
$$

In other words, the node with min-proportional will first access its optimal channel.

The main steps of the DPF algorithm are as follows: at first, SU nodes perceive the CWMN environment and the information of all channels; then, according to processing relational matrixes, we divide the complex $\mathrm{G}$ into some simple graphs. The problem converts into coloring for many simple graphs. Su according to the restrictive conditions of (3) to determine whether access their optimal channel in simple graph. If a node accesses a channel, relational matrix elements must update. Otherwise, wait for next determining. According to the above algorithm, allocate channels until resource depleted or node's needs are satisfied.

\section{SiMULATION AND PERFORMANCE ANALYSIS}

In this section, we evaluate the performance of three algorithms: Parallel Algorithm (short for PA), Maximal Independent Set Algorithm (short for MIS) and our Demand Proportion Fair Algorithm. We consider the grid topology where the grid size is $5 \times 5$ and the distance between adjacent grid points is $100 \mathrm{~m}$. There are $10 \mathrm{SU}$ and $4 \mathrm{PU}$ sharing M 
channel while $M$ alternated from five to twenty-five. We assume the node's coverage radius of transmit power is 1.5. Two nodes cannot engage in successful transmission at the same time if they transmit on the same channel, when the distance between them less then 1.5. In our simulation, we assume the PU would randomly trigger working status and access channel. Then the Su who conflict with the active PU will loss the rewards of related channels.

We get an average of 1000 random experiment data as simulation results. Detailed simulation parameters show in table 1 .

TABLE I. SIMULATION PARAMETERS.

\begin{tabular}{ccc}
\hline Parameter Name & Symbol & Value \\
channel bandwidth & band $_{m}$ & $1 \mathrm{MHz}$ \\
error bit rate & $\mathrm{K}$ & 0.2831 \\
noise power & $\sigma_{0}^{2}$ & $10^{-16} \mathrm{~W} / \mathrm{Hz}$ \\
transmit power & $r_{n, m}$ & $10 \mathrm{mw}$ \\
channel gain & $\mathrm{H}$ & $10^{-5}$ \\
\hline
\end{tabular}

We specially consider the two indicators for comparison purpose: Fairness, the average Throughput of the system.

The throughput under the known interference constraint condition can describe as below:

$$
\text { Throughput }=\frac{1}{N} \sum_{\mathrm{n}=1}^{\mathrm{N}} \theta_{n}
$$

In particular, to compare the fairness, we introduce the maximum proportion fair metric:

$$
\text { Fairness }=\left(\prod_{n=1}^{N} \sum_{m=1}^{M}\left(a_{n, m} \times b_{n, m}\right)+10^{-4}\right)^{\frac{1}{N}}
$$

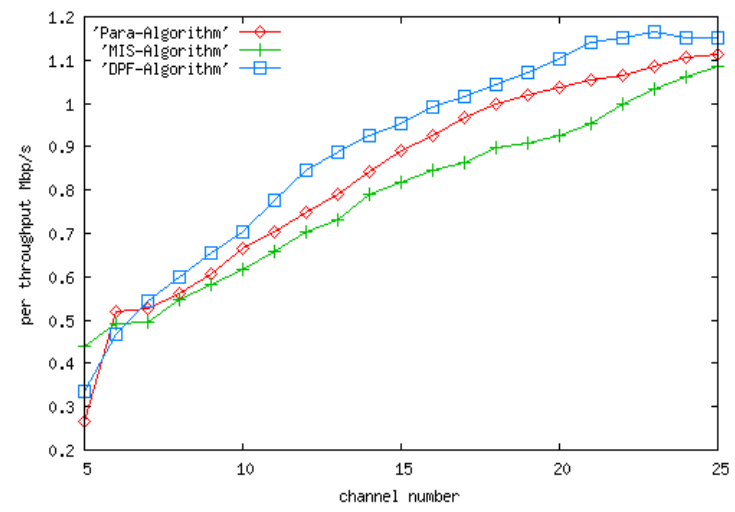

Figure 1. Simulation resulted of throughput

According to figure 1, the throughput of the three algorithms under their own criteria has all shown an uptrend with the increase in the number of channels. Hereinto, the DPF algorithm obtained relatively stable throughput growth by selecting the channel of high speed and long idle time, while the spectrum efficiency of SU in other two algorithms is lower due to the randomly returned collision of PU. When the number of channels increases to a saturated figure, the throughput of three algorithms tends to come together. The results demonstrated in simulation prove that DPF achieves as much as $20 \%$ (even more) improvement in fairness when compared to PA and as about $10 \%$ when compared to MIS. As shown in figure 2, due to the constraint of the introduction of the scale factor, the fairness of DPF is superior to the two other algorithms, and it also has improved the satisfaction of each user' need.

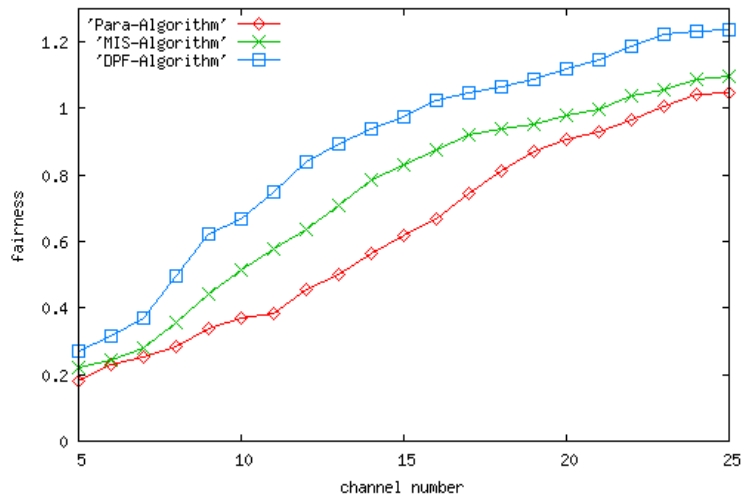

Figure 2. Simulation resulted of fairness

\section{CONCLUSION}

After the text edit has been completed, the paper is ready for the template. Duplicate the template file by using the Save As command, and use the naming convention prescribed by your conference for the name of your paper. In this newly created file, highlight all of the contents and import your prepared text file. You are now ready to style your paper; use the scroll down window on the left of the MS Word Formatting toolbar.

This paper presents a fair channel allocation algorithm based on the needs of users, assesses the channel status and carries out the selective access, with the idle probability density of authorized band and the transmission rate as reference indicators. Meanwhile, the proportion constraint of SU' actual flow resources and demand is set in consideration of the node flow convergence of cognitive mesh network. The simulation results show that the algorithm avoids collision and thus protect the network throughput; at the same time, it improves the fairness and satisfaction of the nodes. However, this paper only considered relatively simple communication situations, the joint optimization of power control and scheduling will be the direction of future researches.

\section{REFERENCES}

[1] Mitola J, Maguire, G Q, Jr 1999. "Cognitive radio making software radios more personal". IEEE Personal Communications. 4 13-8

[2] Lee H, Han K, Hwang Y, Choi S, et al. 1999. "Opportunistic band sharing for point- to- point link connection of cognitive radios". IEEE Cognitive Radio Oriented Wireless Networks and Communications. 1-6

[3] Hiraga K, Akabane K, Shiba H, et al. 2008. "Channel assignment and reallocation algorithms for cognitive radio systems". IEEE Proc of the 14 th Asia-Pacific Conference on Communications. 1-4 
[4] Ak D, Hmk A, Viiayakumar R, Roy S, 2005. "Optimization Models for Fixed Channel Assig nment in Wireles Mesh Networks with Multiple Radios". IEEE Sensor and Ad Hoc Communications and Networks. $463-74$

[5] Wang W, Liu X, 2005. "List-coloring based channel allocation for openspectrum wireless networks". IEEE Vehicular Technology Conference. 690-4

[6] Peng CY, Zheng HT, Zhao BY, 2006 J. "Utilization and fairness in spectrum assignment for opportunistic spectrum access". Mobile Networks and Applications. 4 555-76

[7] Liao CL, Chen J, Tang YX, $2007 \mathrm{~J}$. "Cognitive radio spectrum allocation algorithm in parallel" .Journal of Ectronics \& Information. 7 $1608-11$,
[8] Liu YT, Jiang MX, Tan XZ, Fan L, 2009. "Maximal Independent Set Based Channel Allocation Algorithm in Cognitive Radios".IEEE Youth Conference on Information, Computing and Telecommunication. $78-81$

[9] KIM H, SHIN K G, 2009."Adaptive MAC-layer sensing of spectrum availability in cognitive radio networks" .IEEE. http://www.eecs.umich.edu.cn

[10] YANG L, CAO L, Zheng H, $2008 \mathrm{~J}$. "Proactive channel access in dynamic spectrum netwroks". Physical Communication. 2 103-11

[11] Salem N B, Hubaux J P, 2006. "Securing wireless Mesh networks". IEEE Wireless Communications. 13 50-5 